\title{
Efficiency of Electromagnetic Emission by Electrostatic Turbulence in Solar Wind and Coronal Plasmas with Density Inhomogeneities
}

\author{
A. S. Volokitin ${ }^{1}$ and C. $\mathrm{Krafft}^{2,3}$ (i) \\ ${ }^{1}$ IZMIRAN, Troitsk, 142190, Moscow, Russia \\ ${ }^{2}$ Laboratoire de Physique des Plasmas, Ecole Polytechnique, Institut Polytechnique de Paris, F-91128 Palaiseau Cedex, France \\ ${ }^{3}$ Université Paris-Saclay, Sorbonne Université, Observatoire de Paris, PSL Research University, France; catherine.krafft@u-psud.fr \\ Received 2019 December 21; revised 2020 February 10; accepted 2020 February 11; published 2020 April 24
}

\begin{abstract}
We present a new method to semianalytically calculate the radiation efficiency of electromagnetic waves emitted at specific frequencies by electrostatic wave turbulence in solar wind and coronal plasmas with random density fluctuations. This method is applied to the case of electromagnetic emission radiated at the fundamental plasma frequency $\omega_{p}$ by beam-driven Langmuir wave turbulence during Type III solar bursts. It is supposed that the main radiation mechanism is the linear conversion of electrostatic to electromagnetic waves on the background plasma density fluctuations, at constant frequency. The radiation efficiency (emissivity) of such a process is larger than that obtained in the framework of models where the low frequency density fluctuations and the corresponding ion sound waves are not external but produced by the electrostatic wave turbulence itself through nonlinear wavewave interactions. Results show that the radiation efficiency of Langmuir wave turbulence into electromagnetic emissions at $\omega_{p}$ is nearly constant asymptotically, with the electromagnetic energy density growing linearly with time, and is proportional to the average level of density fluctuations. Comparisons with another analytical method developed by the authors and with space observations are satisfactory.
\end{abstract}

Unified Astronomy Thesaurus concepts: Radio bursts (1339); Solar flares (1496); Solar physics (1476); Plasma physics (2089); Solar wind (1534); Solar corona (1483); Space plasmas (1544); Solar radio emission (1522); Plasma astrophysics (1261); Heliosphere (711); Radio sources (1358)

\section{Introduction}

Electromagnetic radiation associated with Type III solar radio bursts has been observed for a long time both by groundbased radio telescopes and on board spacecraft (e.g., Bougeret et al. 1970; Lin 1974; Fitzenreiter et al. 1976; Gurnett \& Anderson 1977; Steinberg et al. 1984; Suzuki \& Dulk 1985; Lin et al. 1986; Reiner et al. 1992; Benz et al. 2007). It is believed that these radio bursts' emissions at the electron plasma frequency $\omega_{p}$ and its harmonic $2 \omega_{p}$ are the result of a chain of events in which electron flows accelerated during solar flares are generated and emit Langmuir wave turbulence via beam instability during their propagation in the coronal and solar wind plasmas up to distances of 1 au from the Sun and beyond (Zheleznyakov \& Zaitsev 1970; Grognard 1975; Goldman 1983; Mel'nik et al. 1999). In turn, the beam-driven Langmuir waves release part of their energy to electromagnetic radiation. Various mechanisms have been proposed to explain such energy transfer from electrostatic waves to electromagnetic ones. Among the most important, it is worth mentioning the processes of nonlinear wave interactions (Ginzburg \& Zheleznyakov 1958; Melrose 1980), including the decay of Langmuir waves $\mathcal{L}$ into ion sound waves $\mathcal{S}$ and transverse electromagnetic waves $\mathcal{T}_{\omega_{p}}$ of frequency $\omega_{p}$ $\left(\mathcal{L} \rightarrow \mathcal{S}+\mathcal{T}_{\omega_{p}}\right)$, or the fusion of $\mathcal{L}$ and $\mathcal{S}$ waves along the channel $\mathcal{L}+\mathcal{S} \rightarrow \mathcal{T}_{\omega_{p}}$. Moreover, it is believed that the electromagnetic emissions $\mathcal{T}_{2 \omega_{p}}$ at $2 \omega_{p}$ result from the coalescence $\mathcal{L}+\mathcal{L}^{\prime} \rightarrow \mathcal{T}_{2 \omega_{p}}$ of two Langmuir waves $\mathcal{L}$ and $\mathcal{L}^{\prime}$, which are driven by the beam and produced by the electrostatic decay $\mathcal{L} \rightarrow \mathcal{L}^{\prime}+\mathcal{S}$, respectively. Typically, these processes were considered in the framework of the weak turbulence theory. However, in some works, the electromagnetic radiation at $\omega_{p}$ was studied using the strong turbulence theory
(Papadopoulos et al. 1974), assuming that Langmuir wave fields captured in collapsing cavitons and Langmuir solitons can emit such waves (Galeev \& Krasnosel'skikh 1976; Goldman et al. 1980); numerical simulations performed in the strong turbulence regime confirmed the main features of these emissions (Akimoto et al. 1988).

Using realistic Langmuir and ion sound waves' spectra, the theory of weak turbulence allowed for analytical calculations of the rates of electromagnetic emissions generated at $\omega_{p}$ by the wave decay $\mathcal{L} \rightarrow \mathcal{S}+\mathcal{T}_{\omega_{p}}$ (Edney \& Robinson 1999). In another work (Willes et al. 1996), a similar method was used to analytically estimate the radiation rate corresponding to the coalescence process $\mathcal{L}+\mathcal{L}^{\prime} \rightarrow \mathcal{T}_{2 \omega_{p}}$. Moreover, numerical simulations conducted in the framework of weak turbulence (e.g., Kasaba et al. 2001; Li et al. 2009; Li \& Cairns 2013; Ratcliffe et al. 2014) reproduced part of the characteristic features of the dynamics of the observed emission spectra.

Alternative approaches were based on linear conversion mechanisms (Field 1956) of Langmuir or z-mode waves to electromagnetic radiation in plasmas with density gradients. Various aspects of such processes were considered by several authors (Melrose 1980; Cairns \& Willes 2005; Yu \& Kim 2013; Schleyer et al. 2014); in particular, it was shown that such mechanisms may explain the radio emissions by Type III solar bursts (Thejappa et al. 1993). The effectiveness of linear conversion mechanisms was also investigated using numerical methods (Cairns \& Willes 2005; Kim et al. 2008).

On the other hand, it was found that the solar wind plasma is characterized by a large variety of density fluctuations (Celnikier et al. 1983; Kellogg \& Horbury 2005). For example, satellites' measurements revealed that density fluctuations $\delta n$ around several percent of the average background plasma density $n_{0}$ exist in the solar wind, with length scales around a 
few hundreds of kilometers (Mugundhan et al. 2017; Chen et al. 2018; Krupar et al. 2018). These fluctuations, even when weak, affect the development of the electron beam instability and of the intensity and the spectra of the emerging Langmuir wave turbulence (Volokitin et al. 2013; Krafft et al. 2013, 2014, 2015, 2019; Krafft \& Volokitin 2014, 2016b; Voshchepynets et al. 2017). In particular, it was shown (Krafft et al. 2015; Krafft \& Volokitin 2016a) that the electrostatic decay $\mathcal{L} \rightarrow \mathcal{L}^{\prime}+\mathcal{S}$ is less efficient and more localized in space and time in a plasma with density fluctuations than in a uniform plasma. Thus, one can reasonably assume that under such conditions other nonlinear wave processes such as the coalescence $\mathcal{L}+\mathcal{L}^{\prime} \rightarrow \mathcal{T}_{2 \omega_{p}}$ or the decay $\mathcal{L} \rightarrow \mathcal{S}+\mathcal{T}_{\omega_{p}}$ can be weakened in a plasma with inhomogeneities.

Moreover one should note that, as the emissivity of electrostatic wave turbulence into electromagnetic radiation is proportional to the spectral electric field energy and density fluctuation intensity $\left|\delta n_{k} / n_{0}\right|^{2}$, the linear mechanism of wave conversion on external density inhomogeneities is more efficient to generate electromagnetic radiation than the nonlinear wave-wave interaction processes proposed in the frame of the weak turbulence theory. In the latter case the density fluctuations are much weaker (at least by one order of magnitude) because they are induced and depend on the wave turbulence itself; then the corresponding radiation efficiency has a nonlinear dependence on the Langmuir spectral energy, explaining why the wave conversion mechanism leads to larger emissivities (note that the turbulence parameters considered are weak). Moreover, external density fluctuations cannot be neglected in solar wind plasmas, so that a realistic theoretical description cannot avoid taking into account interactions of waves with such density structures. Finally, in the framework of the weak turbulence model, not all small amplitude ion sound waves forming the spectrum $\left|\delta n_{k} / n_{0}\right|^{2}$ can satisfy the resonance conditions required to produce electromagnetic waves through resonant wave-wave nonlinear interactions.

In view of this, the attention to the mechanism of linear transformation of waves on density gradients has increased. Moreover, in solar wind and coronal plasmas, the presence of fluctuating density irregularities leads to the multiple appearance of boundaries between plasma regions of different densities where wave transformations such as refraction, reflection, tunneling, and conversion phenomena occur. Then, using an original approach and realistic wave and density fluctuations' spectra, some authors (Krasnoselskikh et al. 2019) calculated the conversion efficiency of Langmuir waves and obtained estimates in good agreement with the observations. Recently, a new method of determination of the radiation efficiency of electrostatic wave turbulence was proposed (Volokitin \& Krafft 2018), which is based on the direct calculation of the electromagnetic fields emitted at large distances by a plasma source with high-frequency electric currents oscillating at the plasma frequency, determined using a model of Langmuir wave turbulence in a plasma with external background density fluctuations. The semianalytical calculation of the radiation efficiency showed a satisfactory agreement with space observations.

In this paper we present another new semianalytical method of calculation of the efficiency of electromagnetic wave radiation at the frequency $\omega_{p}$ from a plasma with Langmuir wave turbulence and random density fluctuations. As in a previous work (Volokitin \& Krafft 2018), it is assumed that the main mechanism responsible for the generation of electromagnetic waves is the linear conversion of electrostatic waves on the external density fluctuations. Unlike the theory of weak turbulence for a homogeneous plasma, the assumption of weak correlations between the waves is not necessary and, since the electromagnetic emissions do not arise from three-waves' resonant interactions but result from the transformations of Langmuir waves on the density fluctuations, we do not need to take into account exact resonance conditions between the waves.

\section{Electromagnetic Wave Radiation by a Turbulent Plasma with Random Density Fluctuations}

In a previous paper (Volokitin \& Krafft 2018) we calculated the radiation efficiency at far distances of a plasma source with external random density fluctuations and Langmuir wave turbulence emitting electromagnetic waves. The two-dimensional high-frequency current oscillating at the plasma frequency $\omega_{p}$ within the source was calculated owing to the Zakharov equations. Then, by solving an inhomogeneous Klein-Gordon equation involving this current and derived from the Maxwell equations with the help of Green functions, and by using a modified theory of retarded potentials, the electromagnetic energy density emitted at $\omega_{p}$ and the corresponding radiation efficiency were determined as a function of the average level of density fluctuations $\Delta N=\left\langle\left(\delta n / n_{0}\right)^{2}\right\rangle^{1 / 2}$, the ratio $c / v_{T}\left(v_{T}\right.$ is the plasma thermal velocity), and the position of the observer with respect to the source. This method provided radiation efficiencies that were in good agreement with satellite observations of Type III radio bursts' emissions.

Hereafter we present a new and more effective semianalytical method aimed at calculating the radiation efficiency of electrostatic wave turbulence. Let us consider a solar wind plasma source involving Langmuir turbulence and external density fluctuations of average level $\Delta N \simeq 0.01-0.06$, characterized by wavelengths of several hundreds of electron Debye lengths $\lambda_{D}$. These waves scatter on the random density inhomogeneities and can be linearly converted into electromagnetic waves at a constant frequency close to $\omega_{p}$.

Indeed, due to the presence of external density fluctuations, the dominant process responsible for the radiation of electromagnetic waves $\mathcal{T}_{\omega_{p}}$ at the frequency $\omega_{p}$ is the linear conversion of Langmuir waves $\mathcal{L}$ on the inhomogeneities (Denisov 1957; Stix 1965; Cairns \& Willes 2005; Kim et al. 2008; Schleyer et al. 2014), and not the nonlinear wave-wave resonant interactions $\mathcal{L} \pm \mathcal{S} \rightarrow \mathcal{T}_{\omega_{p}}$ involving ion sound waves $\mathcal{S}$ corresponding to induced and weak ion density perturbations. The transformation of Langmuir waves on the external density fluctuations produce an electronic current of the first order whose rotational part is responsible for the radiation of electromagnetic waves as a result of refraction phenomena occurring when the electrostatic waves interact with the density fluctuations. The dynamics of this current can be calculated by solving the Zakharov equations on a 2D map representing the inhomogeneous solar wind plasma. All phenomena of reflection, refraction, and tunneling of waves are taken into account in the simulations. The initial levels of the Langmuir waves' and fluctuations' spectra are chosen according to realistic conditions; their profiles take into account possible anisotropies and the expected wavevector ranges, inferred from results obtained during one-dimensional studies performed by the authors (Volokitin et al. 2013; Krafft et al. 2013, 2014, 2015, 2019; Krafft \& Volokitin 2014, 2016b; Voshchepynets et al. 2017). The 
rotational part of the calculated electronic current generates the induced electromagnetic wave fields as a result of the conversion, at constant frequency, of the electrostatic wave turbulence. The produced waves are then leaving the volume with density fluctuations where they are generated and propagate freely and without loss through the surrounding homogeneous plasma. The model allows us to calculate the emissivity of this first-order wave conversion process owing to numerical simulations confirmed by analytical estimates, as shown below.

The equation describing the electromagnetic waves' dynamics can be obtained from the Maxwell equations

$$
\left(\frac{1}{c^{2}} \frac{\partial^{2}}{\partial t^{2}}+\frac{\omega_{p}^{2}}{c^{2}}-\nabla^{2}\right) \delta \boldsymbol{B}=\frac{4 \pi}{c} \nabla \times \delta \boldsymbol{j},
$$

where $\delta \boldsymbol{B}$ is the wave's magnetic field perturbation. The highfrequency electronic current $\delta \boldsymbol{j}$ produced by the scattering of Langmuir waves on the external density fluctuations is

$$
\delta \boldsymbol{j} \simeq-e \delta n \boldsymbol{v}_{e} \simeq-\frac{i e^{2}}{m_{e} \omega_{p}} \operatorname{Re}\left(\delta n \nabla \tilde{\varphi} e^{-i \omega_{p} t}\right),
$$

where $\delta n$ is the slowly varying electron density perturbation and $v_{e}$ is the fast velocity oscillation (at $\omega_{p}$ ) of the electron population; $e<0$ and $m_{e}$ are the electron charge and mass. The Langmuir wave's potential perturbation is given by $\delta \varphi=\operatorname{Re}\left(\tilde{\varphi} e^{-i \omega_{p} t}\right)$, where $\tilde{\varphi}$ is the slowly varying potential envelope. Note that $\delta \boldsymbol{j}$ is a first-order term as the density fluctuations $\delta n$ are given at the initial state and not induced during the system's dynamics. Then we get

$$
\left(\frac{1}{c^{2}} \frac{\partial^{2}}{\partial t^{2}}+\frac{\omega_{p}^{2}}{c^{2}}-\nabla^{2}\right) \delta \boldsymbol{B} \simeq-\frac{i \omega_{p}}{c} \operatorname{Re}\left(e^{-i \omega_{p} t} \nabla \frac{\delta n}{n_{0}} \times \nabla \tilde{\varphi}\right),
$$

where $n_{0}$ is the background plasma density and $\delta n=\operatorname{Re}\left(\delta \widetilde{n} e^{-i \omega_{s} t}\right) \simeq \operatorname{Re}(\delta \widetilde{n}) ; \omega_{s} \ll \omega_{p}$ is the ion acoustic frequency. Assuming that $\delta \boldsymbol{B}=\operatorname{Re}\left(\tilde{\boldsymbol{B}} e^{-i \omega_{k} t}\right)$, where $\omega_{\boldsymbol{k}} \simeq \omega_{p}$ is the frequency of the electromagnetic wave and $\tilde{\boldsymbol{B}}$ is the slowly varying envelope of its magnetic field, we find that

$$
\left(i \frac{\partial}{\partial t}+\frac{c^{2} \nabla^{2}}{2 \omega_{p}}\right) \tilde{\boldsymbol{B}} \simeq i \frac{c \omega_{p}}{2 \omega_{\boldsymbol{k}}}\left(\nabla \frac{\delta n}{n_{0}} \times \nabla \tilde{\varphi}\right)
$$

where we used that $\left|\partial^{2} \tilde{\boldsymbol{B}} / \partial t^{2}\right| \ll \omega_{k}|\partial \tilde{\boldsymbol{B}} / \partial t|$. Equation (4) can be written in the wavevector space as

$$
\left(i \frac{\partial}{\partial t}-\frac{c^{2} \boldsymbol{k}^{2}}{2 \omega_{p}}\right) \tilde{\boldsymbol{B}}_{\boldsymbol{k}}=i \frac{c \omega_{p}}{2 \omega_{\boldsymbol{k}}}\left(\nabla \frac{\delta n}{n_{0}} \times \nabla \tilde{\varphi}\right)_{\boldsymbol{k}},
$$

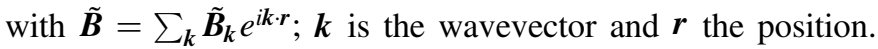
Note that the right-hand side term of Equation (5), proportional to the current density in the plasma source, is calculated by solving at each time moment the 2D Zakharov equations (Volokitin \& Krafft 2018). The homogeneous equation corresponding to Equation (5) can be solved exactly as follows

$$
\tilde{\boldsymbol{B}}_{\boldsymbol{k}}(t)=\tilde{\boldsymbol{B}}_{\boldsymbol{k}}(0) \exp \left(-i \frac{c^{2} \boldsymbol{k}^{2}}{2 \omega_{p}} t\right) \text {. }
$$

A particular solution of Equation (5) is obtained in the form

$$
\begin{aligned}
\tilde{\boldsymbol{B}}_{\boldsymbol{k}}(t)= & \frac{c \omega_{p}}{2 \omega_{\boldsymbol{k}}} \int_{0}^{t}\left(\nabla \frac{\delta n\left(t^{\prime}\right)}{n_{0}} \times \nabla \tilde{\varphi}\left(t^{\prime}\right)\right)_{\boldsymbol{k}} \\
& \times \exp \left(i \frac{c^{2} \boldsymbol{k}^{2}}{2 \omega_{p}}\left(t^{\prime}-t\right)\right) d t^{\prime} .
\end{aligned}
$$

Combining the solutions (6)-(7), we obtain the Fourier component of the magnetic field envelope at time $t$

$$
\begin{aligned}
\tilde{\boldsymbol{B}}_{\boldsymbol{k}}(t)= & \exp \left(-i \frac{c^{2} \boldsymbol{k}^{2}}{2 \omega_{p}} t\right) \\
& \times\left(\tilde{\boldsymbol{B}}_{\boldsymbol{k}}(0)+\frac{c \omega_{p}}{2 \omega_{k}} \int_{0}^{t}\left(\nabla \frac{\delta n\left(t^{\prime}\right)}{n_{0}} \times \nabla \tilde{\varphi}\left(t^{\prime}\right)\right)_{\boldsymbol{k}}\right. \\
& \left.\times \exp \left(i \frac{c^{2} \boldsymbol{k}^{2}}{2 \omega_{p}} t^{\prime}\right) d t^{\prime}\right) .
\end{aligned}
$$

For sufficiently large $|\boldsymbol{k}|$ above a threshold $\left|\boldsymbol{k}_{*}\right|$, the function $\exp \left(i c^{2} \boldsymbol{k}^{2} / 2 \omega_{p} t^{\prime}\right)$ oscillates quickly. Therefore, the main contribution to the integral in Equation (8) is due to the long-wavelength part (with $|\boldsymbol{k}|<\left|\boldsymbol{k}_{*}\right|$ ) of the term $\left(\nabla \frac{\delta n}{n_{0}} \times \nabla \tilde{\varphi}\right)_{\boldsymbol{k}}$. As the simulations performed to calculate $\delta n$ and $\tilde{\varphi}$ from the 2D Zakharov equations show, this term slowly changes with time, so that it can be extracted from the integral. On the other hand, we note that, for $|\boldsymbol{k}| \leqslant\left|\boldsymbol{k}_{*}\right|$, the oscillation rates $\dot{\theta}_{k}$ of the plasmon phases $\theta_{k}$ turn out to be important and comparable with $c^{2} \boldsymbol{k}_{*}^{2} / 2 \omega_{p}$; therefore, we distinguish the phases explicitly from the amplitudes by setting below $\tilde{\varphi}_{\boldsymbol{k}}(t)=\tilde{\varphi}_{\boldsymbol{k}}(0) e^{i \theta_{\boldsymbol{k}}(t)}$. Moreover, one can write that

$$
\left(\boldsymbol{\nabla} \frac{\delta n(t)}{n_{0}} \times \nabla \tilde{\varphi}(t)\right)_{\boldsymbol{k}}=-\sum_{\boldsymbol{k}=\boldsymbol{k}_{1}+\boldsymbol{k}_{2}}\left(\boldsymbol{k}_{1} \times \boldsymbol{k}_{2}\right) \frac{\delta n_{\boldsymbol{k}_{1}}(t)}{n_{0}} \tilde{\varphi}_{\boldsymbol{k}_{2}}(t),
$$

where $\boldsymbol{k}_{1}$ and $\boldsymbol{k}_{2}$ are the wavevectors corresponding to the Fourier components of the density perturbations and the Langmuir wave potential, respectively. Then we get from Equation (8) that

$$
\begin{aligned}
\tilde{\boldsymbol{B}}_{\boldsymbol{k}}(t) \simeq & \exp \left(-i \frac{c^{2} \boldsymbol{k}^{2}}{2 \omega_{p}} t\right) \\
& \times\left(\tilde{\boldsymbol{B}}_{\boldsymbol{k}}(0)-\frac{c \omega_{p}}{2 \omega_{\boldsymbol{k}}} \sum_{\boldsymbol{k}=\boldsymbol{k}_{1}+\boldsymbol{k}_{2}}\left(\boldsymbol{k}_{1} \times \boldsymbol{k}_{2}\right)\right. \\
& \left.\times \int_{0}^{t} \frac{\delta n_{\boldsymbol{k}_{1}}\left(t^{\prime}\right)}{n_{0}} \tilde{\varphi}_{\boldsymbol{k}_{2}}\left(t^{\prime}\right) e^{-i\left(\omega_{\boldsymbol{k}_{1}}^{s}+\omega_{\boldsymbol{k}_{2}}^{l}-\omega_{\boldsymbol{k}}^{t}\right) t^{\prime}} d t^{\prime}\right) .
\end{aligned}
$$

We took into account the dispersion relation of the transverse electromagnetic wave in a homogeneous plasma, i.e., $\omega_{k}^{t}=$ $\left(c^{2} \boldsymbol{k}^{2}+\omega_{p}^{2}\right)^{1 / 2} \simeq \omega_{p}\left(1+c^{2} \boldsymbol{k}^{2} / 2 \omega_{p}^{2}\right)$, so that $c^{2} \boldsymbol{k}^{2} / 2 \omega_{p} \simeq$ $\omega_{\boldsymbol{k}}^{t}-\omega_{p} \simeq \omega_{\boldsymbol{k}}^{t}-\omega_{\boldsymbol{k}_{2}}^{l}-\omega_{\boldsymbol{k}_{1}}^{s}$, where the Langmuir wave frequency is $\omega_{\boldsymbol{k}_{2}}^{l} \simeq \omega_{p}\left(1+3 \lambda_{D}^{2} \boldsymbol{k}_{2}^{2} / 2\right) \simeq \omega_{p}$, with $3 \boldsymbol{k}_{2}^{2} v_{T}^{2} / 2 \ll$ $c^{2} \boldsymbol{k}^{2} ; \omega_{\boldsymbol{k}_{1}}^{s} \ll \omega_{p}$ is the ion sound frequency; as mentioned above, the main contribution to the electromagnetic emission at $\omega_{\boldsymbol{k}}^{t} \simeq \omega_{p}$ in the summation $\sum_{\boldsymbol{k}=\boldsymbol{k}_{1}+\boldsymbol{k}_{2}}$ comes from the domain of small wavevectors satisfying $\boldsymbol{k}^{2} \ll 2 \omega_{p}^{2} / c^{2}$. Note that since we 
are not considering here parametric resonant interactions between waves, but their transformations on density fluctuations, we do not need to fulfill exactly the three-waves' resonance condition $\omega_{\boldsymbol{k}}^{t}=\omega_{\boldsymbol{k}_{2}}^{l}+\omega_{\boldsymbol{k}_{1}}^{s}$. Let us mention that Equation (10) can be compared with the expressions providing the growth rate of the energy density of an electromagnetic wave $\mathcal{T}\left(\omega_{\boldsymbol{k}}^{t}, \boldsymbol{k}\right)$ produced by the fusion $\mathcal{L}+\mathcal{S} \rightarrow \mathcal{T}$ of a Langmuir wave $\mathcal{L}\left(\omega_{\boldsymbol{k}_{2}}^{l}, \boldsymbol{k}_{2}\right)$ and an ion sound wave $\mathcal{S}\left(\omega_{\boldsymbol{k}_{1}}^{s}, \boldsymbol{k}_{1}\right)$, in the frame of the weak turbulence theory (Melrose 1987; Edney \& Robinson 1999).

By raising Equation (8) squared and neglecting the terms proportional to the initial wave magnetic field $\boldsymbol{B}_{\boldsymbol{k}}(0)$, whose amplitude is around the noise level (note that these terms vanish also after the average performed below), we get

$$
\begin{gathered}
\left|\boldsymbol{B}_{\boldsymbol{k}}(t)\right|^{2}-\left|\boldsymbol{B}_{\boldsymbol{k}}(0)\right|^{2} \simeq\left(\frac{c \omega_{p}}{2 \omega_{\boldsymbol{k}}}\right)^{2} \mid \sum_{\boldsymbol{k}=\boldsymbol{k}_{1}+\boldsymbol{k}_{2}}\left(\boldsymbol{k}_{1} \times \boldsymbol{k}_{2}\right) \\
\quad \times\left.\frac{\delta n_{\boldsymbol{k}_{1}}}{n_{0}} \tilde{\varphi}_{\boldsymbol{k}_{2}} \int_{0}^{t} e^{i \theta_{\boldsymbol{k}_{2}}\left(t^{\prime}\right)-i\left(\omega_{\boldsymbol{k}_{1}}^{s}+\omega_{\boldsymbol{k}_{2}}^{l}-\omega_{\boldsymbol{k}}^{t}\right) t^{\prime}} d t^{\prime}\right|^{2}
\end{gathered}
$$

where we took into account that the potential's amplitude varies much more slowly than its phase. The wavevectors of the electromagnetic waves satisfying $|\boldsymbol{k}| \ll\left|\boldsymbol{k}_{1,2}\right|$, the spatial synchronism condition $\boldsymbol{k}_{1}=\boldsymbol{k}-\boldsymbol{k}_{2}$ can be written as $\boldsymbol{k}_{1} \simeq-\boldsymbol{k}_{2}$, so that Equation (11) can be presented in the following form

$$
\begin{aligned}
& \left|\boldsymbol{B}_{\boldsymbol{k}}(t)\right|^{2}-\left|\boldsymbol{B}_{\boldsymbol{k}}(0)\right|^{2} \simeq\left(\frac{c \omega_{p}}{2 \omega_{\boldsymbol{k}}}\right)^{2} \sum_{\boldsymbol{k}_{1}, \boldsymbol{k}_{3}}\left(\boldsymbol{k}_{1} \times \boldsymbol{k}\right) \\
& \cdot\left(\boldsymbol{k}_{3} \times \boldsymbol{k}\right) \frac{\delta n_{\boldsymbol{k}_{1}}}{n_{0}} \frac{\delta n_{\boldsymbol{k}_{3}}^{*}}{n_{0}} \tilde{\varphi}_{-\boldsymbol{k}_{1}} \tilde{\varphi}_{-\boldsymbol{k}_{3}}^{*} \times \int_{0}^{t} e^{i \theta_{\boldsymbol{k}_{3}}+i \frac{c^{2} \boldsymbol{k}^{2}}{2 \omega_{p}} t^{\prime \prime}} \\
& d t^{\prime \prime} \int_{0}^{t} e^{-i \theta_{\boldsymbol{k}_{1}}-i \frac{c^{2} \boldsymbol{k}^{2}}{2 \omega_{p}} t^{\prime}} d t^{\prime}
\end{aligned}
$$

where the superscript "** denotes the complex conjugate. Assuming the absence of correlations between the density fluctuations, let us average on the phases

$$
\left\langle\frac{\delta n_{\boldsymbol{k}_{1}}}{n_{0}} \frac{\delta n_{\boldsymbol{k}_{3}}^{*}}{n_{0}}\right\rangle=\left|\frac{\delta n_{\boldsymbol{k}_{1}}}{n_{0}}\right|^{2} \delta\left(\boldsymbol{k}_{1}-\boldsymbol{k}_{3}\right)
$$

and simplify further Equation (12)

$$
\begin{gathered}
\left|\boldsymbol{B}_{\boldsymbol{k}}(t)\right|^{2}-\left|\boldsymbol{B}_{\boldsymbol{k}}(0)\right|^{2} \simeq\left(\frac{c \omega_{p}}{2 \omega_{\boldsymbol{k}}}\right)^{2} \sum_{\boldsymbol{k}_{1}}\left(\boldsymbol{k} \times \boldsymbol{k}_{1}\right)^{2} \\
\quad \times\left|\frac{\delta n_{\boldsymbol{k}_{1}}}{n_{0}}\right|^{2}\left|\tilde{\varphi}_{\boldsymbol{k}_{1}}\right|^{2}\left|\int_{0}^{t} e^{i \theta_{\boldsymbol{k}_{1}}+i \frac{c^{2} \boldsymbol{k}^{2}}{2 \omega_{p}} t^{\prime}} d t^{\prime}\right|^{2} .
\end{gathered}
$$

Let us now show that asymptotically the following relation is fulfilled

$$
I_{\boldsymbol{k}}=\left|\int_{0}^{t} e^{i \theta_{\boldsymbol{k}_{1}+i \frac{c^{2} \boldsymbol{k}^{2}}{2 \omega_{p}} t^{\prime}}} d t^{\prime}\right|^{2}=\frac{t}{\omega_{p}} \zeta\left(\boldsymbol{k}_{1}, \boldsymbol{k}\right),
$$

where the dimensionless function $\zeta\left(\boldsymbol{k}_{1}, \boldsymbol{k}\right)$ is estimated below. If it is possible to neglect the variations of the plasmons' phases $\theta_{\boldsymbol{k}_{1}}(t)$ in Equation (14), i.e., if $\left|\theta_{\boldsymbol{k}_{1}}\right| \ll\left(c^{2} \boldsymbol{k}^{2} / 2 \omega_{p}\right) t$ for sufficiently large $|\boldsymbol{k}|>\left|\boldsymbol{k}_{*}\right|$, then $I_{\boldsymbol{k}}$ does not depend on $\boldsymbol{k}_{1}$ and is asymptotically proportional to the Dirac function $\delta\left(\boldsymbol{k}^{2}\right)$. Indeed, summing Equation (13) on $\boldsymbol{k}$, its right-hand side term becomes of the form

$$
\begin{aligned}
& \int F\left(\boldsymbol{k}^{2}\right)\left|\int_{0}^{t} e^{i \frac{c^{2} \boldsymbol{k}^{2}}{2 \omega_{p}} t^{\prime}} d t^{\prime}\right|^{2} d \boldsymbol{k}^{2} \\
& =\int F\left(\boldsymbol{k}^{2}\right) \frac{\sin ^{2}\left(\frac{c^{2} \boldsymbol{k}^{2}}{4 \omega_{p}} t\right)}{\left(\frac{c^{2} \boldsymbol{k}^{2}}{4 \omega_{p}}\right)^{2}} d \boldsymbol{k}^{2} \stackrel{t \rightarrow \infty}{\rightarrow} \frac{4 \pi \omega_{p} t}{c^{2}} F(0),
\end{aligned}
$$

where $F\left(\boldsymbol{k}^{2}\right)$ is a continuous function. It follows that, asymptotically, the electromagnetic wave energy density $\sum_{\boldsymbol{k}}\left|\boldsymbol{B}_{\boldsymbol{k}}(t)\right|^{2}$ (13) increases linearly with time within a narrow region of wavevectors near $\boldsymbol{k} \simeq 0$, whereas the size of this region, where electromagnetic waves are generated, decreases with time. This compression stops when the size reaches a value for which the phase $\theta_{\boldsymbol{k}_{1}}(t)$ begins to exceed $\left(c^{2} \boldsymbol{k}^{2} / 2 \omega_{p}\right) t$. Therefore we consider below the case of small $\boldsymbol{k}^{2}$ for which $\left(c^{2} \boldsymbol{k}^{2} / 2 \omega_{p}\right) t \ll\left|\theta_{\boldsymbol{k}_{1}}(t)\right|$, i.e., when the phases $\theta_{\boldsymbol{k}_{1}}(t)$ turn out to be decisive for the calculation of $I_{k}$.

The time dependence of the plasmons' phases is random by nature and determined by their distribution in the inhomogeneous plasma. However, averaging over the random phase variations allows us to state certain conclusions if we assume that $\theta_{\boldsymbol{k}_{1}}=\dot{\theta}_{\boldsymbol{k}_{1}} t$ and that the rates $\dot{\theta}_{\boldsymbol{k}_{1}}$ are randomly distributed. Then we can write that

$$
\left\langle I_{k}\right\rangle=\left\langle\left|\int_{0}^{t} \frac{e^{i \dot{\theta}_{k_{1}} t^{\prime}}}{\dot{\theta}_{\boldsymbol{k}_{1}}} d \dot{\theta}_{\boldsymbol{k}_{1}} t^{\prime}\right|^{2}\right\rangle=\left\langle 4 \frac{\sin ^{2}\left(\theta_{\left.\boldsymbol{k}_{1} / 2\right)}\right.}{\dot{\theta}_{\boldsymbol{k}_{1}}^{2}}\right\rangle .
$$

Assuming that the rates $\dot{\theta}_{\boldsymbol{k}_{1}}$ are uniformly distributed, we get

$$
\begin{aligned}
\left\langle I_{\boldsymbol{k}}\right\rangle & =\int_{\min \dot{\theta}_{\boldsymbol{k}_{1}}}^{\max \dot{\theta}_{\boldsymbol{k}_{1}}} \frac{4 \sin ^{2}\left(\theta_{\boldsymbol{k}_{1}} / 2\right)}{\dot{\theta}_{\boldsymbol{k}_{1}}^{2}} \frac{d \dot{\theta}_{\boldsymbol{k}_{1}}}{\Delta \dot{\theta}_{\boldsymbol{k}_{1}}} \\
& =-\frac{4 t}{\Delta \dot{\theta}_{\boldsymbol{k}_{1}}} \int_{\min \theta_{\boldsymbol{k}_{1}}}^{\max \theta_{\boldsymbol{k}_{1}}} \sin ^{2}\left(\theta_{\boldsymbol{k}_{1}} / 2\right) d\left(\frac{1}{\theta_{\boldsymbol{k}_{1}}}\right) .
\end{aligned}
$$

It follows that the electromagnetic wave energy density $\sum_{k}\left|\boldsymbol{B}_{\boldsymbol{k}}(t)\right|^{2}$ depends linearly on the time $t$. Indeed, for large $t$, the phase interval over which the integration in Equation (17) is performed is large, so that the integral $\int \sin ^{2}\left(\theta_{\boldsymbol{k}_{1}} / 2\right) d\left(1 / \theta_{\boldsymbol{k}_{1}}\right)$ depends very weakly on the boundaries of the variation range $\Delta \dot{\theta}_{\boldsymbol{k}_{1}}=\max \dot{\theta}_{\boldsymbol{k}_{1}}-\min \dot{\theta}_{\boldsymbol{k}_{1}}$. Finally we can conclude that, in the general case, $\zeta\left(\boldsymbol{k}_{1}, \boldsymbol{k}\right)$ is a function that decreases sharply for $|\boldsymbol{k}|>\left|\boldsymbol{k}_{*}\right|$ and mainly depends on $\Delta \dot{\theta}_{\boldsymbol{k}_{1}}$ for $|\boldsymbol{k}|<\left|\boldsymbol{k}_{*}\right|$. Unfortunately, at this stage, it is only possible to estimate the order of magnitude of $\left|\boldsymbol{k}_{*}\right|$ and $\Delta \dot{\theta}_{\boldsymbol{k}_{1}}$. Since both quantities are due to the presence of density fluctuations, the dispersion of electromagnetic waves allows us to write that $c^{2} \boldsymbol{k}_{*}^{2} / 2 \omega_{p} \propto \Delta N$ and $\Delta \dot{\theta}_{\boldsymbol{k}_{1}} \propto \omega_{p} \Delta N$

In conclusion, this analysis allows us to state that, at asymptotic times, the energy density $\sum_{\boldsymbol{k}}\left|\boldsymbol{B}_{\boldsymbol{k}}(t)\right|^{2}$ of the electromagnetic waves generated by a stationary Langmuir wave turbulence via linear transformation mechanisms on density inhomogeneities increases linearly with time. In actual conditions, when the electromagnetic waves have the ability to freely leave the turbulent source, we can speak of a quasi- 
constant rate of conversion of Langmuir waves' energy into an electromagnetic one. So, we assume that all the energy transformed in a given volume leaves it and propagates without loss outside the source through the external uniform plasma. Then the electromagnetic power radiated is determined by

$$
P_{\text {rad }}=\frac{d}{d t} W_{\mathrm{EM}}=\frac{d}{d t} \sum_{\boldsymbol{k}} \frac{\left|\boldsymbol{B}_{\boldsymbol{k}}(t)\right|^{2}}{4 \pi}=\kappa \omega_{p} W_{L},
$$

where $W_{\mathrm{EM}}=\sum_{\boldsymbol{k}}\left|\boldsymbol{B}_{\boldsymbol{k}}\right|^{2} / 4 \pi$ is the electromagnetic wave energy density and $W_{L}=\sum_{k}\left|\boldsymbol{k} \varphi_{\boldsymbol{k}}(t)\right|^{2} / 4 \pi$ is the Langmuir wave energy density in the volume $V_{\text {rad }}$ of the emitting source. The dimensionless quantity $\kappa$ is the ratio of the energy of electromagnetic waves radiated from a given volume of plasma during the period $\omega_{p}^{-1}$ to the energy of Langmuir waves in the same volume. The following expression can be obtained from the theoretical analysis presented above

$$
\begin{aligned}
& \frac{d}{\omega_{p} d t} \sum_{\boldsymbol{k}}\left|\boldsymbol{B}_{\boldsymbol{k}}(t)\right|^{2}=\sum_{\boldsymbol{k}} \frac{\left|\boldsymbol{B}_{\boldsymbol{k}}(t)\right|^{2}-\left|\boldsymbol{B}_{\boldsymbol{k}}(0)\right|^{2}}{\omega_{p} t} \\
& \simeq \sum_{\boldsymbol{k}} \frac{c^{2} \boldsymbol{k}^{2}}{4 \omega_{p}^{2}} \sum_{\boldsymbol{k}_{1}} \sin ^{2} \vartheta\left|\frac{\delta n_{\boldsymbol{k}_{1}}}{n_{0}}\right|^{2}\left|\boldsymbol{k}_{1} \tilde{\varphi}_{\boldsymbol{k}_{1}}\right|^{2} \zeta\left(\boldsymbol{k}_{1}, \boldsymbol{k}\right),
\end{aligned}
$$

where $\vartheta$ is the angle between the plasmon wavevector $\boldsymbol{k}_{1}$ and the small electromagnetic wavevector $\boldsymbol{k}$. In the case of quasiisotropic wave spectra we can write that $\sin ^{2} \vartheta \simeq 1 / 2$, so that

$$
\kappa \simeq \frac{1}{\sum_{\boldsymbol{k}}\left|\boldsymbol{E}_{\boldsymbol{k}}\right|^{2}} \sum_{\boldsymbol{k}_{1}}\left|\frac{\delta n_{\boldsymbol{k}_{1}}}{n_{0}}\right|^{2}\left|\boldsymbol{E}_{\boldsymbol{k}_{1}}\right|^{2} \sum_{\boldsymbol{k}} \frac{c^{2} \boldsymbol{k}^{2}}{8 \omega_{p}^{2}} \zeta\left(\boldsymbol{k}_{1}, \boldsymbol{k}\right),
$$

where $\left|\boldsymbol{E}_{\boldsymbol{k}_{1}}\right|^{2}=\left|\boldsymbol{k}_{1} \varphi_{\boldsymbol{k}_{1}}\right|^{2}$. As the term $S=\sum_{\boldsymbol{k}} \frac{c^{2} \boldsymbol{k}^{2}}{8 \omega_{p}^{2}} \zeta\left(\boldsymbol{k}_{1}, \boldsymbol{k}\right)$ in Equation (19) is not sufficiently defined at this stage, we have to turn to numerical simulations. Nevertheless, using the estimates $c^{2}\left|\boldsymbol{k}_{*}\right|^{2} / 2 \omega_{p}^{2} \sim \Delta N, \quad \Delta \dot{\theta}_{\boldsymbol{k}_{1}} \sim \omega_{p} \Delta N \quad$ and $\zeta\left(\boldsymbol{k}_{1}, \boldsymbol{k}\right) \sim \omega_{p} / \Delta \dot{\theta}_{k_{1}}$, and omitting possible numerical factors, we can write that $S \sim \sum_{k} \boldsymbol{k}^{2} / \boldsymbol{k}_{*}^{2}$. Thus, one can expect a weak dependence of $S$ on $\Delta N$, or even no dependence, as confirmed by the numerical simulations. In this case, the scaling $\kappa \propto \Delta N$ follows from Equation (19) with

$$
\kappa \simeq \frac{1}{\sum_{\boldsymbol{k}}\left|\boldsymbol{E}_{\boldsymbol{k}}\right|^{2}} \sum_{\boldsymbol{k}_{1}}\left|\frac{\delta n_{\boldsymbol{k}_{1}}}{n_{0}}\right|^{2}\left|\boldsymbol{E}_{\boldsymbol{k}_{1}}\right|^{2} S,
$$

where $S$ can be interpreted as a fraction of phase volume in the $\boldsymbol{k}$-space corresponding to the fraction of plasmons with wavevectors $\boldsymbol{k}_{1}$ transformed on density fluctuations into electromagnetic waves of close wavevectors. Note that, in Equation (20), only the factor $S$ contains a possible dependence on the speed of light $c$; from a formal point of view, one can expect that $S \rightarrow 0$ if $c \rightarrow \infty$. Indeed, the radiation intensity of a localized current source decreases with increasing ratio of the emitted wavelength (proportional to $c$ ) to the size of the source, and tends to zero when $c \rightarrow \infty$. This remains true in the case of linear transformations of electrostatic waves into electromagnetic ones when they scatter on inhomogeneities of finite size.

Let us now compare the analytical results obtained above with those provided by the numerical simulations performed on the basis of a 2D modeling of Langmuir wave turbulence in a plasma with density inhomogeneities (see the description in Volokitin \& Krafft 2018), which allows us to determine the distribution of the high-frequency electronic currents in a given plasma source region. Next, solving Equation (5) numerically or, equivalently, calculating the wave magnetic field using Equation (8), we can find the time evolution of the electromagnetic waves' amplitudes according to the numerical scheme described in the Appendix where, in particular, the necessary restriction on the discretization time step is indicated. It was shown that numerical results are stable under this condition.

The main conclusions provided by the simulations are consistent with those derived analytically above, namely, the linear growth with time, at the asymptotic stage, of the electromagnetic energy density emitted by the turbulent plasma volume. Therefore, it is possible to determine, using the simulation results, the rate of the ratio of the electromagnetic wave energy to the energy of electrostatic Langmuir waves, that is, the radiation efficiency defined according to

$$
\kappa=\frac{d}{\omega_{p} d t}\left(\frac{\sum_{k}\left|\boldsymbol{B}_{k}(t)\right|^{2}}{\sum_{k}\left|\boldsymbol{k} \varphi_{\boldsymbol{k}}(t)\right|^{2}}\right) .
$$

We used the same notation $\kappa$ for the value obtained theoretically in Equation (20) and for the definition of the radiation efficiency presented in Equation (21), as in both cases the physical meaning is the same.

The variations of $\kappa$ as a function of the two characteristic parameters $c / v_{T}$ and $\Delta N$ have been studied $\left(v_{T}\right.$ is the plasma thermal velocity). They were obtained by using Equation (21) with fields computed by the numerical simulations. Note that all variables below are normalized according to $\omega_{p} t \rightarrow t$, $\boldsymbol{r} / \lambda_{D} \rightarrow \boldsymbol{r}$, and $\sqrt{4 \pi n_{0} T_{e}} / E \rightarrow E$, where $T_{e}$ is the electron temperature of the background plasma. All analyses illustrated hereafter by figures were performed within time intervals where wave spectra were quasi-stationary.

Figure 1 shows the electromagnetic wave spectra at asymptotic times, for three values of the ratio $c / v_{T}$ and a fixed average level of density fluctuations $\Delta N$. One can see that, as it should be, the size of the $\boldsymbol{k}$-space where electromagnetic waves are generated does not depend on the time step $\Delta t$ (compare the upper and the bottom panels) and is proportional to $\left(c / v_{T}\right)^{-1}$, so that its area scales as $\left(c / v_{T}\right)^{-2}$. This result is in accordance with the scaling laws presented below in Figure 3. Note also that, at the asymptotic stage, the size of the $\boldsymbol{k}$-space region where the electromagnetic waves' amplitudes grow does not change with time (not shown here), which confirms the conclusions obtained above when analyzing analytically $I_{\boldsymbol{k}}$ (Equations (14)-(17)).

Figure 2 shows the growth with time of the electromagnetic wave energy density $\sum_{k}\left|\boldsymbol{B}_{\boldsymbol{k}}(t)\right|^{2}$ calculated at the asymptotic stage by the numerical simulations; the corresponding linear interpolations describe the time dependence predicted by the analytical calculations well (Equations (14)-(17)). The values of the radiation efficiency $\kappa$ are proportional to the time derivatives of $\sum_{k}\left|\boldsymbol{B}_{\boldsymbol{k}}(t)\right|^{2}(21)$, i.e., to the slopes of the interpolation lines. Note that, due to the statistical oscillations which are natural to the processes considered, the time variations of the derivatives (not shown here) are noticeable compared to the linear approximations. Therefore, in order to avoid such numerical uncertainties during the calculation of $\kappa$, averaging on time was carried out, corresponding to the 

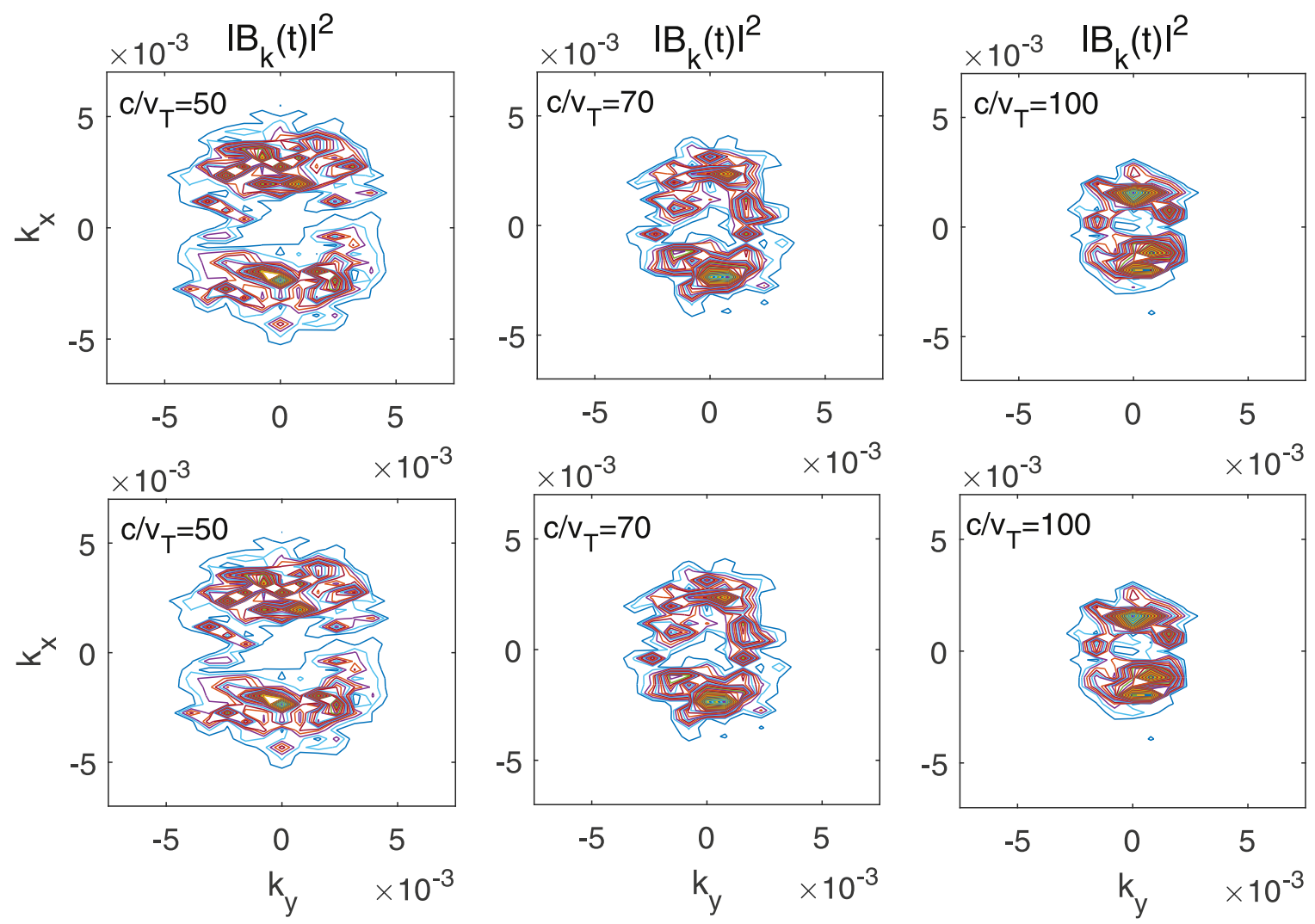

Figure 1. Electromagnetic wave spectra at asymptotic times (around $t \simeq 9800$ ): isocontours of the square spectral wave magnetic field $\left|\boldsymbol{B}_{\boldsymbol{k}}(t)\right|^{2}$, for $c / v_{T}=50,70$, and 100; $\Delta N=0.027 ; k_{x}$ and $k_{y}$ are the normalized wavevectors' components along and across the ambient magnetic field direction, respectively. The time steps used for the fields' calculations (see the Appendix) are $\Delta t=8$ (upper panels) and $\Delta t=2$ (bottom panels).

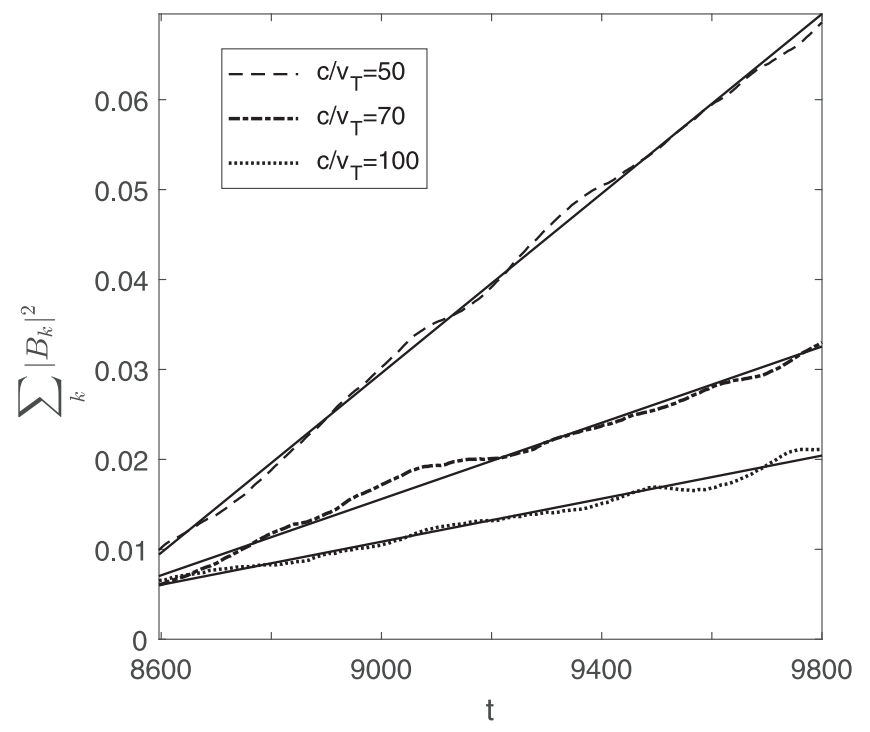

Figure 2. Growth with time, at the asymptotic stage, of the electromagnetic wave energy density $\sum_{k}\left|\boldsymbol{B}_{k}(t)\right|^{2}$ calculated by the numerical simulations (dashed and dotted lines) superposed to their linear interpolations (solid lines). Three values of $c / v_{T}$ are considered : 50, 70, and 100; $\Delta N=0.027$. All variables are normalized.

substitution in Equation (21) of the time derivatives by the slopes of the linear approximations.

The upper panels of Figure 3 show the results of such calculations and present the dependence of the radiation efficiency $\kappa$ as a function of the ratio $c / v_{T}$ and the level of density fluctuations $\Delta N$, respectively. A clear dependence of $\kappa$ on these parameters can be observed, even if its calculated values are more or less scattered. The radiation efficiency is shown to vary linearly with $\Delta N$, as expected from the above analytical developments; moreover, the scaling law $\kappa \propto\left(c / v_{T}\right)^{-2}$ obtained is in good agreement with the results provided by Figure 1 and with the above remarks concerning the behavior of $S(20)$ with increasing $c$.

These results can be compared with those obtained using the numerical simulations and another analytical method presented earlier by the authors (Volokitin \& Krafft 2018), which is based on the theory of the retarded potentials. The corresponding radiation efficiency $\kappa_{r}$ is shown in the bottom panels of Figure 3, as a function of $c / v_{T}$ and $\Delta N$. One can see that, despite some numerical uncertainties inherent to both approaches (compare upper and bottom panels), the values of the radiation efficiencies $\kappa$ and $\kappa_{r}$ provided by the two semianalytical methods are in reasonable agreement. Moreover it is worth noting that the scaling laws $\kappa \propto \Delta N$ and $\kappa \propto\left(c / v_{T}\right)^{-2}$ are obtained using both approaches.

\section{Conclusion}

The electromagnetic radiation at the plasma frequency $\omega_{p}$ generated by a plasma source with electrostatic wave turbulence and density fluctuations is studied theoretically and numerically. It is assumed that the main mechanism of radiation of the electromagnetic emissions is the linear transformation of Langmuir waves on the density inhomogeneities. This process is likely the most effective if the average level of density fluctuations $\Delta N$ is of the order of $1 \%$ of the background plasma density or higher, what is the case for the 

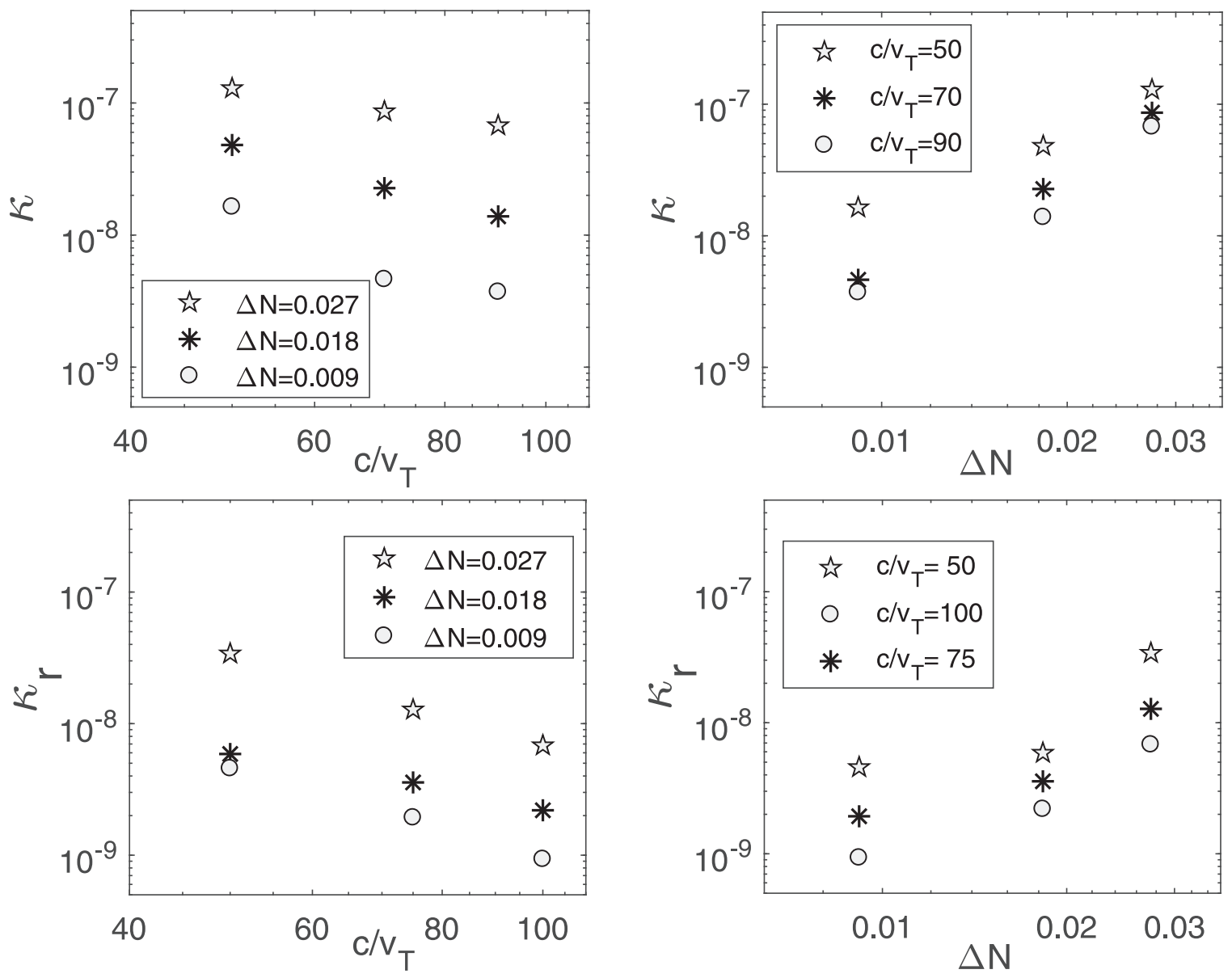

Figure 3. (Upper panels) Variations of the radiation efficiency $\kappa$ calculated using the numerical simulations and Equation (21), as a function of the velocity ratio $c / v_{T}$ (for three values of $\Delta N=0.009,0.018$, and 0.027 , left) and the average level of density fluctuations $\Delta N$ (for three values of $c / v_{T}=50,70$, and 90 , right). The axes' scales are logarithmic. (Bottom panels) Variations of the radiation efficiency $\kappa_{r}$ calculated using the numerical simulations and a method based on the theory of the retarded potentials (Volokitin \& Krafft 2018), as a function of the velocity ratio $c / v_{T}$ (for three values of $\Delta N=0.009,0.018$, and 0.027 , left) and the average level of density fluctuations $\Delta N$ (for three values of $c / v_{T}=50,75$, and 100, right). The axes' scales are logarithmic.

solar wind and coronal plasma regions where Type III solar radio bursts manifest.

We present a new method to calculate analytically the radiation efficiency of electromagnetic waves emitted at some specific frequencies by electrostatic wave turbulence in a plasma with random density fluctuations. This method, which complements another one proposed recently by the authors (Volokitin \& Krafft 2018) is more convenient to use and more robust. Moreover it is shown that, despite significant differences between both approaches, the two methods lead to close values of the radiation efficiencies and provide similar scaling laws as a function of the average level of density fluctuations $\Delta N$ and the velocity ratio $c / v_{T}$.

More precisely, a simple analytical expression is obtained (Equation (20)) that allows us to determine the efficiency of the electromagnetic radiation from a turbulent and inhomogeneous plasma, if the density fluctuations' and the Langmuir waves' spectra are available. In particular, it shows that the radiation efficiency depends mainly on the integral characteristics of the spectra, is weakly sensitive to detail features and is proportional to the average level of density fluctuations $\Delta N$.

When deriving Equation (20), a number of assumptions were made that appear to be sufficiently substantiated. Nevertheless, our theoretical results were verified using numerical simulations based on a 2D modeling of Langmuir wave turbulence in a plasma with quasi-random density inhomogeneities, that confirmed our assumptions.

This work was granted access to the HPC resources of IDRIS under the allocation 2013-i2013057017 made by GENCI. This work has been done within the LABEX Plas@par project, and received financial state aid managed by the Agence Nationale de la Recherche, as part of the programme "Investissements d'avenir" under the reference ANR-11IDEX-0004-02. This work was supported by the Programme National PNST of CNRS/INSU cofunded by CNES and CEA.

\section{Appendix}

Let us present the numerical scheme used to calculate the wave radiation intensity emitted at time $t$ from the $2 \mathrm{D}$ turbulent inhomogeneous plasma source. Starting from Equation (8) that provides the magnetic field's Fourier component $\tilde{\boldsymbol{B}}_{k}(t)$, one can obtain after one time step $\Delta t \ll t$ that

$$
\begin{aligned}
& \tilde{\boldsymbol{B}}_{\boldsymbol{k}}(t+\Delta t) \simeq \exp \left(-i \frac{c^{2} \boldsymbol{k}^{2}}{2 \omega_{p}} \Delta t\right)\left[\tilde{\boldsymbol{B}}_{\boldsymbol{k}}(t)+\frac{c \omega_{p}}{2 \omega_{\boldsymbol{k}}}\right. \\
& \left.\times \int_{0}^{\Delta t}\left(\nabla \frac{\delta n}{n_{0}} \times \nabla \tilde{\varphi}\right)_{\boldsymbol{k}}(t+\tau) \exp \left(i \frac{c^{2} \boldsymbol{k}^{2}}{2 \omega_{p}} \tau\right) d \tau\right],
\end{aligned}
$$


where we defined $\tau=t^{\prime}-t$. Then, expressing the term proportional to the electronic current as follows

$$
\begin{aligned}
\boldsymbol{S}(t+\tau) & =\frac{c \omega_{p}}{2 \omega_{k}}\left(\nabla \frac{\delta n}{n_{0}} \times \nabla \tilde{\varphi}\right)_{k}(t+\tau) \\
& \simeq \boldsymbol{S}(t)+\frac{\boldsymbol{S}(t+\Delta t)-\boldsymbol{S}(t)}{\Delta t} \tau,
\end{aligned}
$$

we get

$$
\begin{aligned}
& \tilde{\boldsymbol{B}}_{\boldsymbol{k}}(t+\Delta t) \simeq \exp \left(-i \frac{c^{2} \boldsymbol{k}^{2}}{2 \omega_{p}} \Delta t\right) \\
& \times\left(\tilde{\boldsymbol{B}}_{\boldsymbol{k}}(t)+\int_{0}^{\Delta t}\left(\boldsymbol{S}(t)+\frac{\boldsymbol{S}(t+\Delta t)-\boldsymbol{S}(t)}{\Delta t} \tau\right)\right. \\
& \left.\times \exp \left(i \frac{c^{2} \boldsymbol{k}^{2}}{2 \omega_{p}} \tau\right) d \tau\right) .
\end{aligned}
$$

The calculation of the integrals in Equation (A2) leads to the rather simple expression

$$
\begin{aligned}
& \tilde{\boldsymbol{B}}_{k}(t+\Delta t) \simeq e^{-i a \Delta t}\left[\tilde{\boldsymbol{B}}_{k}(t)+\left(\frac{e^{i a \Delta t}-1}{i a}\right) \boldsymbol{S}(t)\right. \\
& \left.-\left(\frac{(i a \Delta t-1) e^{i a \Delta t}+1}{a^{2} \Delta t}\right)(\boldsymbol{S}(t+\Delta t)-\boldsymbol{S}(t))\right],
\end{aligned}
$$

where $a=c^{2} \boldsymbol{k}^{2} / 2 \omega_{p}$. Note that in our case, because the electromagnetic emissions come from a very small region of the $\boldsymbol{k}$-space, we do not need to take into account the correlations between the density fluctuations $\delta n / n_{0}$ and the potential's envelope $\tilde{\varphi}$, contrary to what is done in the frame of the weak turbulence theory. A similar formula can be obtained using Equation (5) and finite-difference methods

$$
\begin{aligned}
& \tilde{\boldsymbol{B}}_{\boldsymbol{k}}(t+\Delta t) \simeq\left(\frac{1-i a \Delta t / 2}{1+i a \Delta t / 2}\right) \tilde{\boldsymbol{B}}_{\boldsymbol{k}}(t) \\
& \quad+\frac{c \omega_{p}}{2 \omega_{\boldsymbol{k}}} \frac{\Delta t}{1+i a \Delta t / 2}\left(\nabla \frac{\delta n}{n_{0}} \times \nabla \tilde{\varphi}\right)_{k}(t),
\end{aligned}
$$

which is valid if $a \Delta t / 2=\left(c^{2} \boldsymbol{k}^{2} / 4 \omega_{p}\right) \Delta t \leqslant 1$ and if $\left(\nabla\left(\delta n / n_{0}\right) \times \nabla \tilde{\varphi}\right)_{k}(t)$ varies negligibly during the small time interval $\Delta t$. Then, for $\left(c^{2} \boldsymbol{k}^{2} / 4 \omega_{p}\right) \Delta t \ll 1$, we get the simple approximate formula

$$
\begin{gathered}
\tilde{\boldsymbol{B}}_{\boldsymbol{k}}(t+\Delta t) \simeq\left(1-i \frac{c^{2} \boldsymbol{k}^{2}}{2 \omega_{p}} \Delta t\right) \tilde{\boldsymbol{B}}_{\boldsymbol{k}}(t) \\
+\frac{c \omega_{p}}{2 \omega_{\boldsymbol{k}}} \Delta t\left(\nabla \frac{\delta n}{n_{0}} \times \nabla \tilde{\varphi}\right)_{\boldsymbol{k}}(t) .
\end{gathered}
$$

Note that $\omega_{p} \Delta t=2-10$ in the above study.

Using Equations (A3) or (A4) and knowing the values of $\boldsymbol{S}(t)$ (A1) at any time owing to the 2D simulations based on the Zakharov equations (see Volokitin \& Krafft 2018 for more details), we can calculate numerically $\left|\boldsymbol{B}_{\boldsymbol{k}}(t)\right|^{2}$ as well as the electromagnetic wave energy density $W_{\mathrm{EM}}$ and the radiation efficiency $\kappa$.

\section{ORCID iDs}

\section{Krafft (i) https://orcid.org/0000-0002-8595-4772}

\section{References}

Akimoto, K., Rowland, H. L., \& Papadopoulos, K. 1988, PhFl, 31, 2185 Benz, A. O., Brajša, R., \& Magdalenić, J. 2007, SoPh, 240, 263

Bougeret, J.-L., Caroubalos, C., Mercier, C., \& Pick, M. 1970, A\&A, 6, 406 Cairns, I. H., \& Willes, A. J. 2005, PhPl, 12, 052315

Celnikier, L. M., Harvey, C. C., Jegou, R., Moricet, P., \& Kemp, M. 1983, A\&A, 126, 293

Chen, X., Kontar, E. P., Yu, S., et al. 2018, ApJ, 856, 73

Denisov, N. G. 1957, JETP, 4, 544

Edney, S. D., \& Robinson, P. A. 1999, PhPl, 6, 3799

Field, G. B. 1956, ApJ, 124, 555

Fitzenreiter, R. J., Evans, L. G., \& Lin, R. P. 1976, SoPh, 46, 437

Galeev, A., \& Krasnosel'skikh, V. 1976, JETPL, 24, 515

Ginzburg, V., \& Zheleznyakov, V. 1958, SvA, 2, 653

Goldman, M. V. 1983, SoPh, 89, 403

Goldman, M. V., Reiter, G. F., \& Nicholson, D. R. 1980, PhFl, 23, 388

Grognard, R. J. M. 1975, AuJPh, 28, 731

Gurnett, D. A., \& Anderson, R. R. 1977, JGR, 82, 632

Kasaba, Y., Matsumoto, H., \& Omura, Y. 2001, JGR, 106, 18693

Kellogg, P. J., \& Horbury, T. S. 2005, AnGeo, 23, 3765

Kim, E.-H., Cairns, I. H., \& Robinson, P. A. 2008, PhPl, 15, 102110

Krafft, C., \& Volokitin, A. 2014, EPJD, 68, 370

Krafft, C., \& Volokitin, A. 2016a, in AIP Conf. Proc. 1720, SOLARWIND 14, ed. G. P. Zank et al. (Melville, NY: AIP), 040008

Krafft, C., Volokitin, A., Krasnoselskikh, V., \& Dudok de Wit, T. 2014, JGRA, 119,9369

Krafft, C., \& Volokitin, A. S. 2016b, ApJ, 821, 99

Krafft, C., Volokitin, A. S., \& Gauthier, G. 2019, Fluids, 4, 69

Krafft, C., Volokitin, A. S., \& Krasnoselskikh, V. V. 2013, ApJ, 778, 111

Krafft, C., Volokitin, A. S., \& Krasnoselskikh, V. V. 2015, ApJ, 809, 176

Krasnoselskikh, V., Voshchepynets, A., \& Maksimovic, M. 2019, ApJ, 879, 51

Krupar, V., Maksimovic, M., Kontar, E. P., et al. 2018, ApJ, 857, 82

Li, B., \& Cairns, I. H. 2013, ApJL, 763, L34

Li, B., Cairns, I. H., \& Robinson, P. A. 2009, JGRA, 114, A02104

Lin, R. P. 1974, SSRv, 16, 189

Lin, R. P., Levedahl, W. K., Lotko, W., Gurnett, D. A., \& Scarf, F. L. 1986, ApJ, 308, 954

Mel'nik, V. N., Lapshin, V., \& Kontar, E. 1999, SoPh, 184, 353

Melrose, D. 1987, SoPh, 111, 89

Melrose, D. B. 1980, SoPh, 67, 357

Mugundhan, V., Hariharan, K., \& Ramesh, R. 2017, SoPh, 292, 155

Papadopoulos, K., Goldstein, N. L., \& Smith, R. A. 1974, ApJ, 190, 175

Ratcliffe, H., Kontar, E. P., \& Reid, H. A. S. 2014, A\&A, 572, A111

Reiner, M., Stone, R., \& Fainberg, J. 1992, ApJ, 394, 340

Schleyer, F., Cairns, I. H., \& Kim, E.-H. 2014, JGRA, 119, 3392

Steinberg, J. L., Hoang, S., Lecacheux, A., Aubier, M. G., \& Dulk, G. A. 1984, A\&A, 140, 39

Stix, T. H. 1965, PhRvL, 15, 878

Suzuki, S., \& Dulk, G. A. 1985, in Solar Radiophysics: Studies of Emission from the Sun at Metre Wavelengths, ed. D. J. McLean \& N. R. Labrum (Cambridge: Cambridge Univ. Press), 289

Thejappa, G., Lengyel-Frey, D., Stone, R. G., \& Goldstein, M. L. 1993, ApJ, 416,831

Volokitin, A., Krasnoselskikh, V., Krafft, C., \& Kuznetsov, E. 2013, in AIP Conf. Proc. 1539, SOLARWIND 13, ed. G. P. Zank et al. (Melville, NY: AIP), 78

Volokitin, A. S., \& Krafft, C. 2018, ApJ, 868, 104

Voshchepynets, A., Volokitin, A., Krasnoselskikh, V., \& Krafft, C. 2017, JGRA, 122, 3915

Willes, A. J., Robinson, P. A., \& Melrose, D. B. 1996, PhPl, 3, 149

Yu, D. J., \& Kim, K. 2013, PhPl, 20, 122104

Zheleznyakov, V. V., \& Zaitsev, V. V. 1970, SvA, 14, 47 